\title{
MODELLING OF DOUBLE-PEAKED EMISSION LINES IN AGN'S
}

\author{
L.S.NAZAROVA \\ Royal Greenwich Observatory, Cambridge CB3OEZ, U.K.
}

Optical spectra of the galaxies NGC3227, Mkn6, Mkn9, Mkn279, Mkn376 with double-peaked emission lines are presented. The data were obtained with the 1024pixel TV scanner of the 6-m telescope during the 1984-1991 period. The doublepeaked structure of the $H \beta$ line in most galaxies shows changes during this period and only in the galaxy Mkn6 is the double-peaked stucture of $H \beta$ constant. In all the galaxies except Mkn6, the blue peak of line coincides with the rest-wavelength of the line (the opposite occurs in Mkn6).

It is suggested that the double-peaked line profiles are due to large scale gas motion in the disk envelope which characterizes the radial and the tangential components of velocity. The double-peaked profiles of $H \beta$ have been modelled with an elliptical geometry for the gas envelope of these galaxies. The shape of the envelope in the models is determined by the tangential component of velocity. The equations of stationary population of levels were solved by Sobolev's probabilistic method. The velocity gradient is determined for the envelope with axially symmetric kinematics. It is also suggested that the rotation axis has an arbitrary inclination to the observer. Comparison of the theoretical and observed profiles of the lines shows that the double-peaked structure of $H \beta$ in the galaxies could be produced in the envelope of the accretion disk which is characterised by Keplerian rotation $V_{\theta}=$ const $* R^{-0.5}$ and radial component of velocity $V_{r}=$ const $* R^{-1}$ with decelerated expansion for the galaxies NGC3227, Mkn9, Mkn279, Mkn376 and with accretion for Mkn6.

Key words: data analysis-galaxies: NGC3227, Mkn6, Mkn9, Mkn279, Mkn376-galaxies Seyfert-lines:profile-double-peaked structure

T. J.-L. Courvoisier and A. Blecha: Multi-Wavelength Continuum Emission of AGN, 452. (C) 1994 IAU. Printed in the Netherlands. 